\title{
Apuntes para una educación escolar de la sexualidad. Una lectura de sus fundamentos modernos desde la pedagogía queer ${ }^{1}$
}

Notes for a school education of sexuality. A reading of its modern foundations from the queer pedagogy

\author{
Carolina Ojeda \\ Universidad Nacional de La Plata, Argentina \\ carolina.ojeda.rincon@gmail.com
}

\author{
Pablo Scharagrodsky \\ Universidad Nacional de Quilmes, Argentina \\ pas@unq.edu.ar \\ Santiago Zemaitis \\ Universidad Nacional de La Plata - CONICET, Argentina \\ zemaitis.santiago@gmail.com
}

\section{ReSUMEN:}

En este artículo, nos proponemos una lectura de algunos de los fundamentos modernos de la educación escolar de la sexualidad, desde la lente de la pedagogía queer, rama crítica de la pedagogía contemporánea, que permite poner en cuestión las prácticas educativas en general y, de forma particular, aquellas que, en relación con los géneros y las sexualidades, son producidas y naturalizadas por el discurso escolar. Argumentamos que es necesario -e inevitable- imaginar la posibilidad de deconstruir visiones sobre las identidades, los cuerpos y los placeres, acogiendo la invitación que esta pedagogía nos hace de subvertir un orden pedagógico tradicional, heteronormativo, y por tanto, patriarcal.

Palabras ClaVe: Pedagogía queer, Educación Sexual, Heteronorma.

\section{ABstract:}

In this article we propose reading some of the modern foundations of school education of sexuality, from the lens of queer pedagogy, a critical branch of contemporary pedagogy, which allows to question educational practices in general and in a particular way, those that, related to genders and sexualities, are produced and naturalized by the school discourse. We argue that it is necessary -and inevitable - to imagine the possibility of deconstructing visions about identities, bodies and pleasures, by accepting the invitation that this pedagogy makes us to subvert a traditional pedagogical order, heteronormative, and therefore, patriarchal.

KeYwords: Queer Pedagogy, Sex Education, Heteronorma.

\section{INTRODUCCIÓN}

En este artículo, retomamos algunas discusiones que tuvieron lugar en diferentes encuentros en el marco de un proyecto de extensión universitaria, iniciado en el 2016 -y actualmente en curso- titulado "Prácticas de comunicación y educación por la desobediencia sexo-genérica”. ${ }^{2}$ En los intercambios con estudiantes, las reflexiones giraron en torno a las diferentes ideas, representaciones y opiniones a propósito del lugar de los cuerpos, los géneros y las sexualidades en las escuelas. ${ }^{3}$

Como resultado de dichos intercambios, hemos sistematizado seis problemáticas o tensiones naturalizadas en torno a la educación de la sexualidad que entendemos son posibles de deconstruir en el marco de la pedagogía queer ${ }^{4}$ en diálogo con los estudios de género y la pedagogía feminista. ${ }^{5}$ 
Este ejercicio se despliega en forma de ensayo, inspirado en discusiones suscitadas en diferentes ámbitos. Como rasgo constitutivo de los estudios queer, y algunas corrientes feministas, acudimos a voces que no hacen parte del canon académico, ni necesariamente del ámbito educativo: las calles, la disidencia, el grito, la consigna política, la performance, entre otras expresiones políticas y culturales, todas las cuales nos han permitido operar pedagógicamente sobre los fundamentos modernos de la educación escolar de la sexualidad.

Comenzamos la reflexión presentando algunos puntos de partida con los que organizamos la discusión. La primera de ellas tiene que ver con asumir que los cuerpos no tienen una entidad ontológica, no existen en estado "natural", sino que están insertos en una particular trama de sentidos y significados siempre inestables, móviles y en permanente disputa. Preciado (2014, s/p) sugiere que "no hay cuerpo sino un conjunto heterogéneo, siempre en ruptura, de categorías de conocimiento, sistemas de representación, técnicas de gobierno, que luchan por producir modos de subjetivación". Esto quiere decir que es en el marco de ciertos órdenes discursivos (institucionales, económicos y políticos), que se le asignan al cuerpo, determinados sentidos recurrentemente precarios, fugaces y transitorios. En relación a los discursos, estos configuran sistemáticamente los objetos de los que hablan, es decir, no los definen o identifican sino que los construyen y al hacerlo, ocultan su propia invención (Foucault, 1999; 2002). En otras palabras, al describir un objeto o tema (la sexualidad, el deseo, el placer, las emociones, etc.), los discursos en cierto modo los inventan y los dotan de ciertos sentidos, mientras excluyen u omiten otros posibles, incluso cuando sólo pretendan describirlos tal como son.

En segundo lugar, entendemos la educación escolar de la sexualidad como un campo polisémico de discursos de diverso orden y estatuto (médico, pedagógico, psicológico, psicoanalítico, jurídico, religioso, entre otros) que son articulados, superpuestos o enfrentados en un campo de disputas por sus sentidos y finalidades (Wainerman, Di Virgilio y Chami,2008; Zemaitis, en prensa). Desde allí, lejos de suponer una coherencia entre modelos o en lo que las propuestas institucionales plantean como educación sexual, lo que encontramos es un conjunto variado, heterogéneo y muchas veces contradictorio de objetos, prácticas y políticas que fueron aglutinados bajo ese nombre (Boccardi, 2008). Consideramos, por otro lado, que nuestro horizonte de reflexión se dirige y puede colaborar en potenciar inicialmente a la educación sexual integral (ESI), ${ }^{6}$ como apuesta estatal que acompañamos y defendemos, y que, de cara a sus alcances, así también de sus detractores y las vigentes resistencias, nos está sugiriendo repensarla a 12 años de su promulgación y sumarle otras urgencias y otros modos de ver a los sujetos en formación -niñxs y jóvenes-. En este sentido, creemos que una mirada queer, en tanto ejercicio de interrogación (Trujillo, 2015), puede acaso potenciarla y dar lugar a lecturas y prácticas más conscientes de que toda apuesta educativa, todo marco epistemológico y teórico y todo ejercicio de enseñanza, es siempre un actuar político atravesado por relaciones de poder y/ o de dominación.

Sostenemos, por otro lado, que la escuela como institución moderna, con matices, fugas de sentido y ciertas resistencias, produce y reproduce modernidad sexuada cuyos tonos predominantes son la binariedad, el dimorfismo sexual, la jerarquía sexual y racial, la abyección, cierta proxemia y sensibilidad corporal, sancionando o censurando otras opciones posibles de actuar, pensar y sentir (Lopes Louro, 1997, 1999; Pineau, 2014; Scharagrodsky, 2007). Vale precisar en este punto que por fundamentos modernos nos referiremos a estas categorías y grillas de comprensión (binarismo, jerarquía sexual, etc.) producidas al calor de los cambios culturales, políticos y pedagógicos que supusieron los procesos de secularización y construcción de los Estados nacionales, indicando el fin de la Edad Media. Hacia los finales del siglo XVIII, y en el marco de las revoluciones burguesas (Revolución industrial en Inglaterra y la Revolución Francesa), fue cuando la modernidad como clima de época se instaló definitivamente en las sociedades occidentales (Caruso y Dussel, 1996). ${ }^{7}$ Enfatizamos que estos puntos de partida, si bien han sido expuestos de manera separada, están pensados en relación de interdependencia y afectación constitutiva.

En lo que sigue, entonces, exponemos seis problemas acompañados de ciertos interrogantes que, como anunciamos anteriormente, fueron discutidos en los encuentros de extensión, a saber: 1) La heteronorma 
como matriz de inteligibilidad de la educación sexual o ¿cómo, quiénes y por qué definen una economía de deseo normal?; 2) El cuerpo como dato biológico/fisiológico y universal o ¿qué cuerpo produjo y transmitió la pedagogía moderna?; 3) La homosexualidad como contagio o ¿cómo se habló -y qué se silenció- de la homosexualidad?; 4) La reproducción como único fin de la sexualidad o ¿cómo se define y explica la sexualidad?; 5) La pedagogización de la sexualidad o ¿qué es lo educable en la educación sexual?; 6) La mirada adultocéntrica de la sexualidad o ¿cómo aprendemos a ser niños o niñas? Cada uno de estos problemas e interrogantes enuncian un fundamento moderno, sobre el cual se ha erigido una cierta manera de educar y gestionar la sexualidad. Finalmente, cerramos el trabajo en forma provisoria, con una invitación a cuestionar esos fundamentos y considerar otras alternativas posibles.

\section{LA HETERONORMA COMO MATRIZ DE INTELIGILIDAD DE LA EDUCACIÓN DE LA SEXUALIDAD O ¿CÓMO, QUIÉNES Y POR QUÉ DEFINEN UNA ECONOMÍA DEL DESEO NORMAL?}

Una de las características de la heteronormatividad tiene que ver con referenciar la heterosexualidad como si fuese coherente y otorgarle una posición de privilegio que no puede ser cuestionada (Berlant y Warner, 2002). Sin embargo, para tener realidad 'ontológica', la heterosexualidad necesita de un par opuesto: la homosexualidad. Este binarismo (hetero/homo) es necesario para el funcionamiento de la heteronorma en la medida que la heterosexualidad no se cuestiona ni se visibiliza en tanto tal. Desde allí, quedan por fuera un buen resto de experiencias, deseos e identidades sexuales, ya sea como anormalidad, enfermedad o rareza.

Un ejemplo de esto podemos encontrarlo en el discurso escolar de la educación sexual y no debemos para ello ir muy atrás en el tiempo: en el año 1987, el Ministerio de Educación y Justicia publicó un cuadernillo sobre educación sexual en el que, entre varios otros puntos, se asoció dentro de las "perversiones" a la homosexualidad. En el texto se indicaba: "Perversión es una categoría en la que se incluyen los sujetos que practican la sexualidad de un modo diferente al habitual. Sustituyen preferentemente y a veces condiciones normales del orgasmo o las conductas relacionadas a él (...)" (Ministerio de Educación y Justicia, 1987, s/ p). Según este material, se consideraba una de las clasificaciones de las perversiones la "Deformación de la imagen de la pareja. Ejemplo: zoofilia (objeto sexual: animales); homosexualidad (objeto sexual: individuos del mismo sexo); pedofilia (objeto sexual niños)" (Ministerio de Educación y Justicia, 1987, s/p).

Como se puede observar, una pedagogía fundada en este tipo de enunciados, no solo refuerza la idea de que toda educación sexual debe orientarse a una educación para la heterosexualidad, excluyendo y estigmatizando otras opciones de deseo posibles; ${ }^{8}$ sino también equipara la orientación sexual homosexual con la zoofilia y la pedofilia.

De forma más explícita, estas ideas siguieron vigentes dentro de la órbita del Ministerio de Educación cuando, en el año 1992, se publicaron una serie de artículos sobre educación sexual y en uno de ellos se postulaba que los "Problemas de lo privado a lo público" incluirían "Situaciones existentes que trascendieron a lo público entre las cuales cabe mencionar: crisis de la institución matrimonial, de las vida de familia, conflictos de los vínculos; Homosexualidad/feminismo/gay/travestismo/lesbianismo; Nuevas posibilidades para la «relación de pareja»; Anticoncepción: responsabilidad en maternidad y paternidad; Conocimiento de la Sexualidad y del Amor: posibilidad de la pareja enriquecida de descubrir al amor-placer; Existencia de una madurez psico-afectiva: capacidad de comprometerse de la mujer y el varón que es necesario cultivar y promover (Ministerio de Educación y Justicia, 1992, p. 60).

En este caso, se reforzaba la orientación a la heterosexualidad a partir de la idea moderna de la constitución de la pareja con capacidad reproductiva y siempre en el marco del matrimonio. Amor y pareja, juventud y matrimonio, resultaban ser asociaciones que ficcionalizaban un determinado sujeto al cual estaba destinada la educación sexual: la pareja heterosexual joven, unidos por una determinada manera de conceptualizar el amor. 
Si tomamos en cuenta que estos y otros discursos formaron parte del currículum escolar oficial en la historia reciente, consideramos entonces que el desafío no solo sería revisar este tipo de definiciones moralizantes y normativistas, sino pensar en clave de reivindicar -y agenciar- los placeres. Una muestra de esto que planteamos, se aprecia en algunas de las respuestas pacíficas y creativas a los continuos ataques de homo/ lesbofobia en lugares públicos. En estas, personas de todas las edades y orientaciones convocan a Besatones bajo el lema "Ningún beso nace hetero". Así podemos apreciarlo en las figuras 1, 2 y 3 reproducidas a continuación.

FIGURA 1:

"Ningún beso nace hetero"

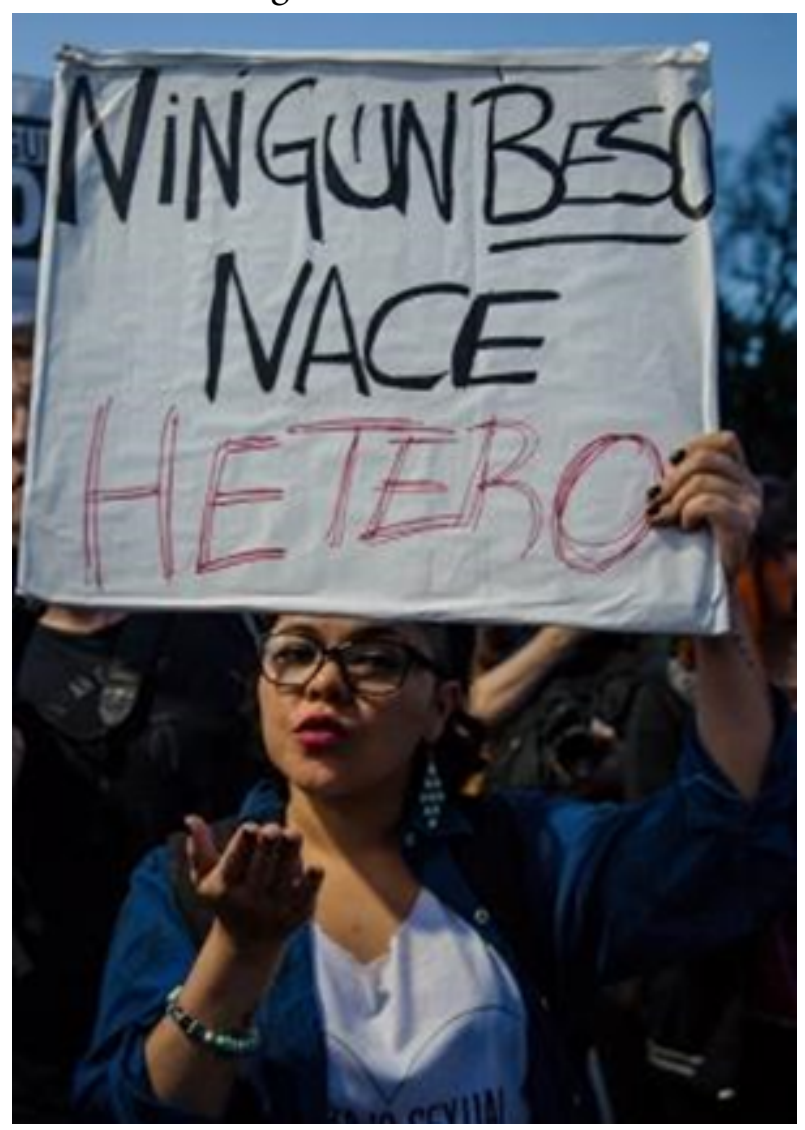

(Magdalena, 2017). 
FIGURA 2:

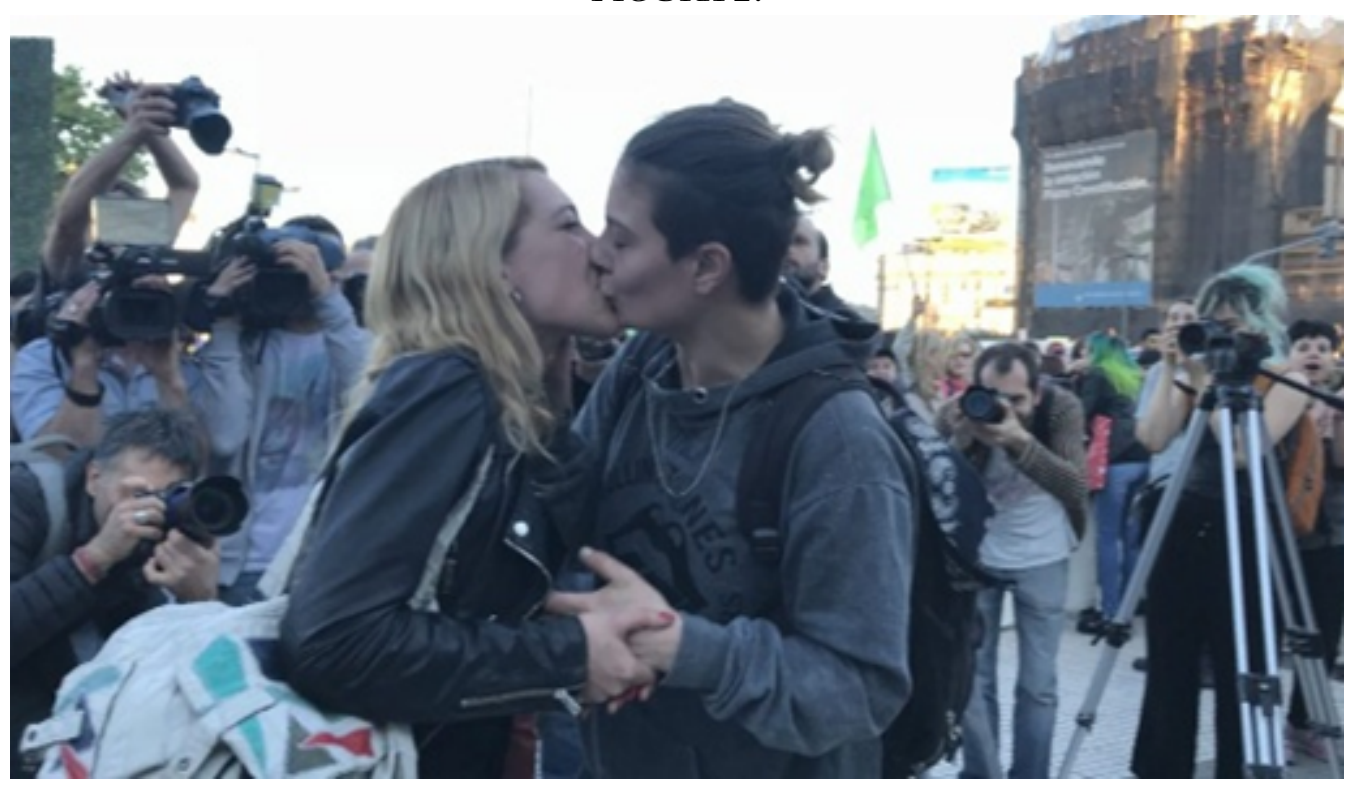

“\#BesosLesbianos Procesan a Mariana Gómez, detenida cuando besaba a su esposa”. Este caso de discriminación y lesbofobia generó movilizaciones y manifestaciones de Tortazo y Besatón en la estación de subte "Constitución” en la ciudad de Buenos Aires en el 2017 (Agencia Presentes, 2017)

FIGURA 3:

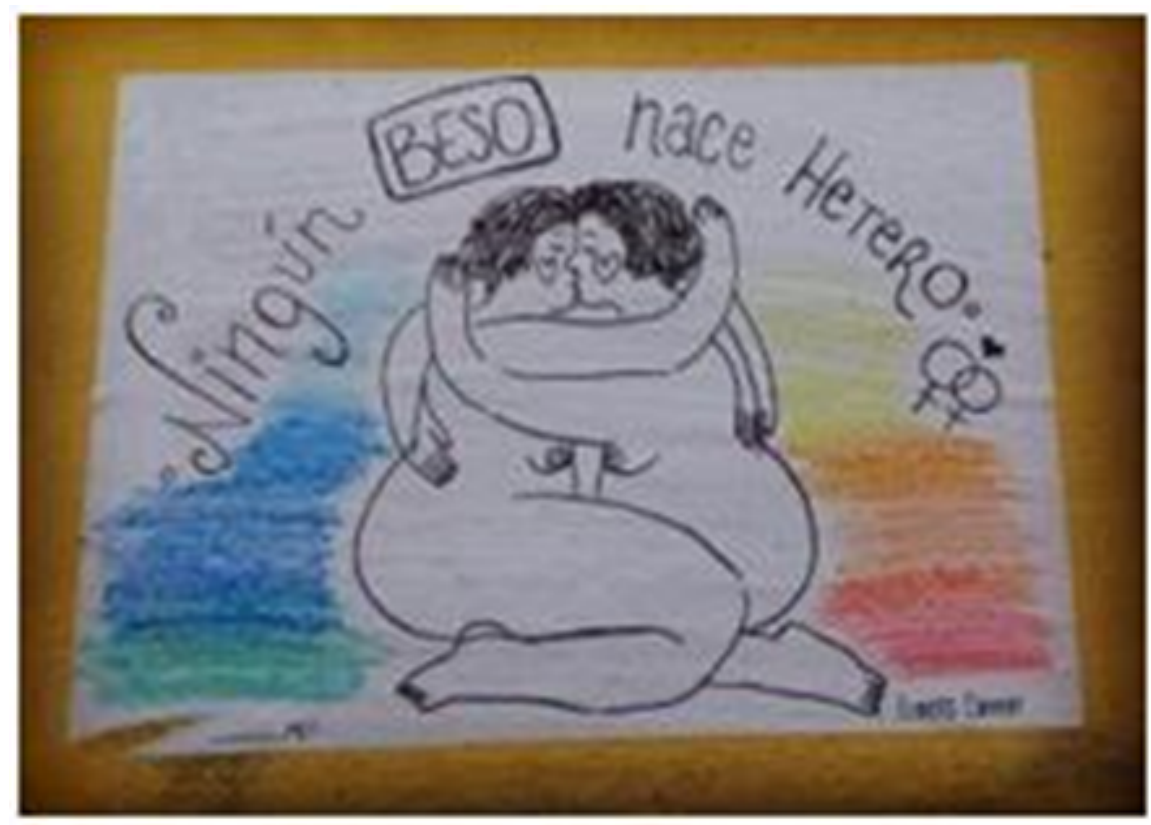

Fotografía tomada por Carolina Ojeda, coautora de este artículo, el 24 de septiembre de 2016. Lugar: Baño del Centro Social y Cultural Olga Vázquez en la ciudad de La Plata

\section{EL CUERPO COMO DATO BIOLÓGICO/FISIOLÓGICO Y UNIVERSAL O ¿QUÉ CUERPO PRODUJO Y TRANSMITIÓ LA PEDAGOGÍA MODERNA?}

La pedagogía moderna produjo un ideal corporal dominante sobre el cual se fundaron doctrinas pedagógicas. Cuerpos blancos, dimórficos, heterosexuales, simétricos, saludables y productivos, obedientes en sus gestos y 
miradas, pulcros y aseados en sus vestimentas, racionales y geométricos en su silueta, jerárquicos en función al deseo, clase social, color de piel, religión, etc. La construcción de este cuerpo ideal se asentó en la lógica de la mismidad, produciendo, a su vez, otredades que la afirmaran ya sea como normal, verdadero o deseable, excluyendo $\mathrm{u}$ omitiendo otras formas posibles de pensar, conceptualizar y, sobre todo, de experimentar la corporalidad.

Son innumerables los ejemplos que podríamos usar para ilustrar este segundo problema ya que, sabemos, no será otra cosa que el cuerpo, aquello que las instituciones modernas intervienen y producen. A comienzos del siglo XX, el Mayor General del ejército británico Robert Baden-Powell fundó el movimiento Scout, uno de los movimientos de educación no formal más importantes de dicho siglo. Si bien esta fue una propuesta que no surgió dentro de la escuela, pronto fue adaptada con el propósito de formar los hombres viriles aptos para los novatos Estados nacionales (Méndez y Scharagrodsky, 2016; Scharagrodsky, 2004). Los principios del scoutismo, abiertamente militares e imperialistas, propugnaban por la defensa nacional, la exploración y el dominio de la naturaleza, y el rechazo a la vida urbana que se asumía decadente y amenazante, a partir de la virilización de los cuerpos, inicialmente cuerpos masculinos. En este sentido, no solo afirmaba una identidad masculina blanca (Hantover, 1978), sino también, restituía la distinción de los roles sexuales, que se veía amenazada con la expansión de ciertas ideas feministas (Badinter, 1993). En tanto que propuesta corporal, su surgimiento en el contexto de la conquista colonial tendrá "el objetivo de mantener el poder racial sobre las personas colonizadas" (Connell, 1998, p. 162). Desde allí, observamos la producción de jerarquías somáticas.

La relación con la naturaleza en términos de su dominación y el fortalecimiento de la fraternidad masculina tenían su otredad: Tomasito, el joven "pie tierno", cuyo cuerpo débil, frágil y temeroso, constituía aquello que un boyscout tendría que evitar o rechazar ser. Esto puede apreciarse tanto en la figura 4 como en la figura 5 que vemos a paso siguiente:

FIGURA 4.

Cap 2: En campaña (p. 58).

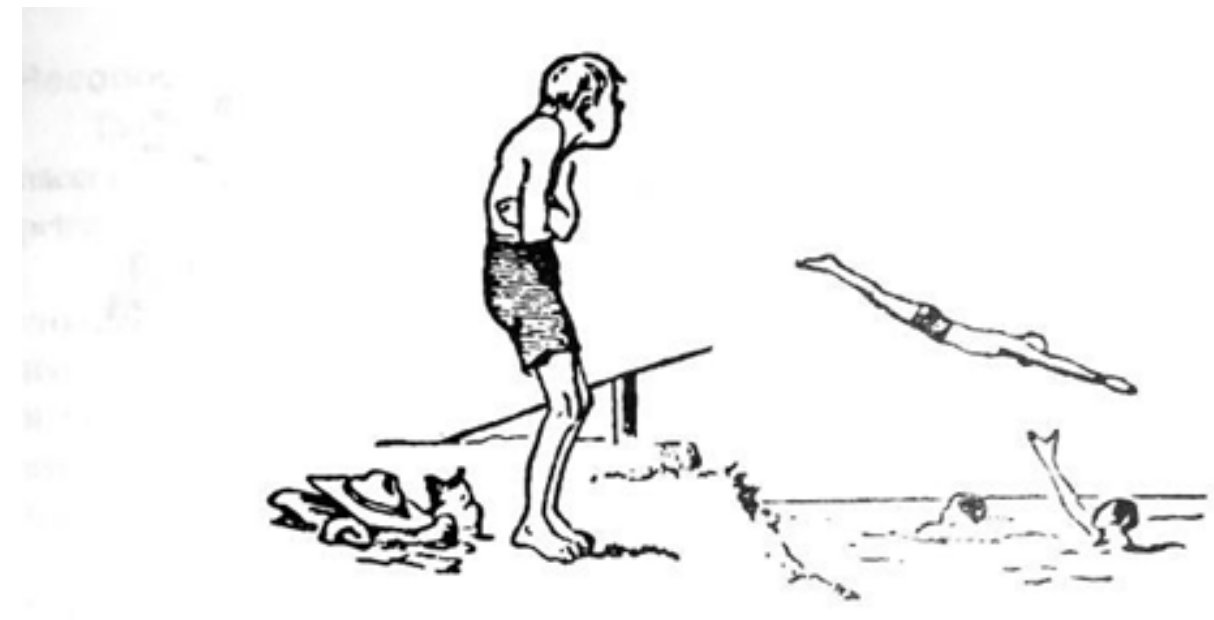

TOMASITO, EL PIE TIERNO. $N^{\circ} 1$

TOMASITO EN EL LAGO

Tomasito ve a todos felices, pues se echan al agua y saben nadar, pero él, infeliz. no sabe y no se puede echar. 
FIGURA 5.

Cap 3: Vida de campamento (p. 84)

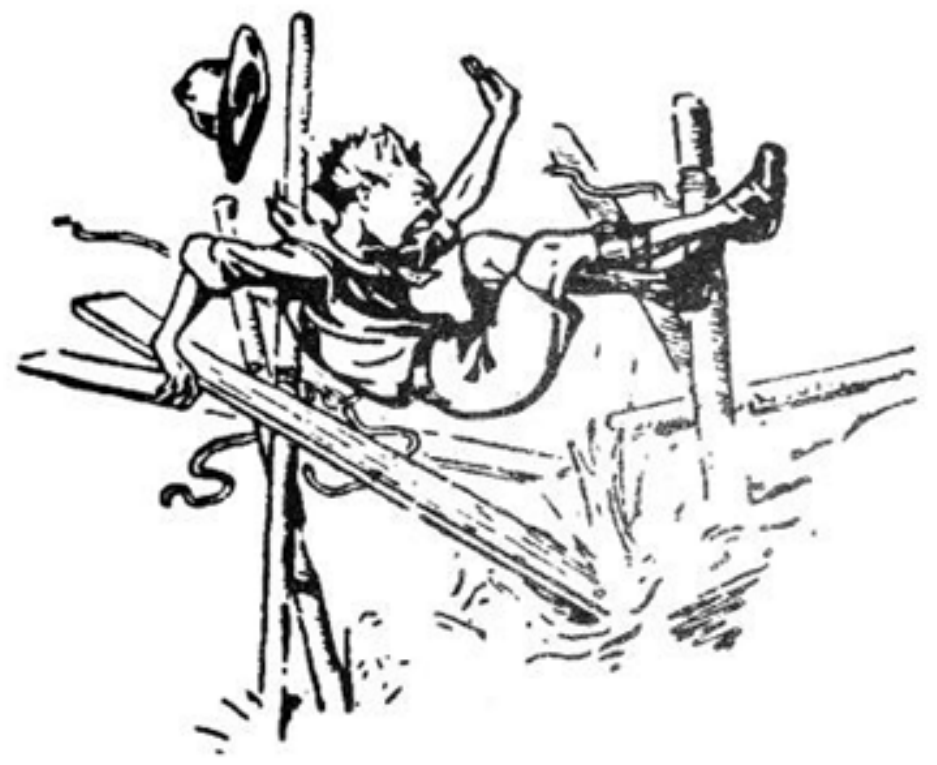

TOMASITO, EL PIE TIERNO. $N^{\circ} 3$
TOMASITO CONSTRUYE UN PUENTE

Vuestros nudos son los que dan resistencia a vuestro puente. Los nudos de Tomasito han arruinado el suyo.

Baden-Powell(1998 [1908]). Escultismo para muchachos.

¿Cuántos Tomasitos han habitado y habitan hoy el espacio escolar? ¿Qué asignaturas y contenidos se encargan de sostener y naturalizar Tomasitos como otredades? ¿De qué maneras asignaturas como la educación física reproducen un canon corporal sexualizado, racializado y generizado?, y por otro lado, ¿Qué cualidades tiene Tomasito que beneficiarían el espacio escolar? ¿Acaso no vendría bien resistirse a competir, conquistar o dominar? Estas preguntas ayudan a problematizar el canon corporal de la pedagogía moderna, aquel que excluye Tomasitos y pretende definir los límites de los cuerpos. Por ello, desde las disidencias, gritamos con Diana Torres (2011, p. 11):

Mi coño, mi polla, mis orificios todos, mi orgasmo:

donde he construido un monumento al deseo que siempre está lubricado.

(...) Mi cuerpo, mi cuerpo, MI CUERPO.

Donde yo mando, ¡cabrones!

4. La homosexualidad como contagio o ¿Cómo SE Habló y QUÉ SE SILENCIÓ DE LA HOMOSEXUALIDAD? ${ }^{9}$

El arbitrario régimen de la heterosexualidad (Wittig, 2006), circunscribe una determinada forma de concebir la sexualidad, el deseo y los placeres, y opera más allá de los importantes cambios legislativos y las políticas de las ltimas décadas. ${ }^{10} \mathrm{Al}$ hacerlo, excluye otras alternativas posibles de pensar, experimentar y vivir la sexualidad. Consolidar el imperativo heterosexual, implica ciertas identificaciones sexuadas, determinando cuáles son los cuerpos viables o posibles y, al mismo tiempo, excluyendo y repudiando las prácticas e identidades homoeróticas. 
Desde la fundación misma de los sistemas educativos modernos, los pedagogos confiaban en que el despertar sexual debía ser tempranamente controlado, vigilado y regulado en relación al posible contagio del homosexualismo. Este interés pone de relieve un aspecto que evidencia que el discurso pedagógico moderno ha atribuido a lo sexual un poder sustantivo, vinculándolo a la formación moral, intelectual y física. La educación diferenciada entre alumnos varones y alumnas mujeres correspondía a un interés por la educación de la (hetero)sexualidad, como sugiere Louise Georges Tin, 2012, p. 20): “estas diferenciaciones pedagógicas tenían como objetivo reinstaurar cierta diferenciación (sexual) en medio de la escolaridad mixta (heterosexual). Se trataba de lograr que la escolaridad mixta (de los sexos) no desembocara en la confusión (de los géneros)".

Así, la escuela moderna, en su formato pedagógico con sus discursos disciplinares, se ha erigido sobre una matriz de pensamiento heterosexual y sexista resultando ser uno de los mecanismos sociales más importantes y potentes que regularía la distribución de rasgos y características diferenciales y desiguales a varones y a mujeres conformando un 'orden corporal escolar' generizado y heterosexualizado a partir de la delimitación de ciertas reglas, prácticas y saberes (Scharagrodsky, 2007).

Una de las referencias pedagógicas argentinas más destacadas en los inicios del siglo XX fue Víctor Mercante. Su impronta y perspectivas se materializaron en la cultura normalista y sus obras se pueden ubicar en el marco de los desarrollos científicos que junto con otros pedagogos de su época, contribuyeron a moldear la formación de las generaciones siguientes de educadores y psicólogos, -y de parte de la opinión pública a través de la prensa de divulgación- consolidando un modo específico de definir problemas educativos e imaginar resoluciones desde ese campo (Draghi y Vassiliades, 2014; Talak, 2010). A propósito de las escuelas y conventos de niñas, el pedagogo Mercante postulaba que era desde los 13 años "cuando el amor ofrece esas extrañas perversiones conocidas bajo el nombre de homosexualismos" (Mercante, (2013 [1918]), p. 147). Estas se daban, según él, bajo la forma de "amistades solitarias", en un principio, para luego convertirse en una "emoción fiamesca", ${ }^{11}$ y por último, se podría resolver en "uranismo". ${ }^{12}$

Actualmente, no solo es anacrónico y violento identificar experiencias lesbianas y homosexuales con perversión y contagio -aun cuando voces de los grupos conservadores y de ultraderechas así lo sigan sosteniendo-, sino que resulta repudiable en relación a los avances legislativos y culturales sobre la aceptación de las orientaciones no heterosexuales, como elecciones legítimas del deseo. Entonces, una pedagogía escolar de la sexualidad, tendrá que situarse en contra de todo discurso o práctica que represente un posicionamiento homo/lesbofóbico, o que muestre las manifestaciones del deseo como "casos" o "problemas". Esto es, no evitar o silenciar las situaciones de discriminación o violencia hacia personas que no tengan un deseo heterosexual, sea cual fuere el modo de autoadscripción genérico-sexual. Una pedagogía de la sexualidad abierta y en línea con las transformaciones actuales, supera los resabios de los viejos modelos de la educación sexual instalados desde la modernidad, como el moralista o las visiones sanitaristas o del sexo seguro (Jones, 2009), que han colaborado activamente en sostener y legitimar perspectivas patologizantes y normalizadoras del deseo.

Fue precisamente pensando en las generaciones futuras que Lemebel (1986) nos interpeló hace unos años con su escritura punzante, con la que hoy dialogamos reflexionando el daño que, como señala flores (2016), conlleva todo proceso de normalización sexo-genérica, y que bien puede verse como marca constitutiva de los cuerpos. Desde allí, Lemebel (1986) sigue más vigente que nunca:

Hay tantos niños que van a nacer

Con una alita rota

Y yo quiero que vuelen compañero

Que su revolución

Les dé un pedazo de cielo rojo

Para que puedan volar 


\section{LA REPRODUCCIÓN COMO ÚNICO FIN DE LA SEXUALIDAD O ¿CÓMO SE DEFINIÓ Y EXPLICÓ LA SEXUALIDAD?}

Históricamente, en la enseñanza de las formas de reproducción de la vida (sexual, asexual), en la descripción de los órganos, gónadas y hormonas internas de ambos sexos (siempre solo dos sexos), se erigieron como equivalentes los términos "sexualidad", "órganos reproductivos/sexuales", "reproducción biológica”. Esta tradición en la enseanza moderna de las ciencias naturales ha enfatizado nicamente la dimensión más material de la sexualidad: el cuerpo y la definición de los órganos genitales.

En una gran variedad de manuales y textos escolares, estos fueron llamados únicamente como "reproductivos" colocando la reproducción de la especie como su única finalidad. Los mismos, también, se los suele nombrar como "sexuales", siendo las únicas partes referenciadas en tanto tales (sistema reproductor masculino/sistema reproductor femenino), como si otras zonas de los cuerpos no generaran placer, o bien, suponiendo que no existen otros usos eróticos del cuerpo por fuera de los sistemas y órganos reproductivos. Adjetivar como "sexuales" determinados órganos también es una operación con voluntad de delimitar y organizar las zonas legítimas de placer del cuerpo, una suerte de jerarquía sexuada de la superficie corporal (Zemaitis, 2016).

En este sentido, Preciado (2009) plantea que en la escuela se continúa con el proceso de exclusión y jerarquización de las partes del cuerpo así como con la asignación normalizada de funciones: mano para escribir, boca para comer, órganos sexuales para la reproducción, etc. Desde allí, los cuerpos, sus formas y funciones, los usos que les damos, son definidos desde una matriz heterosexual aparentemente invisible.

La historia de la educación sexual nos ofrece innumerables ejemplos al respecto. El modelo biologicista moderno, que tuvo vigencia durante gran parte del siglo $\mathrm{XX}$, centró la enseñanza de la sexualidad a sus aspectos anatomofisiológicos. Desde este planteamiento, queda cristalizada una forma específica sobre la sexualidad: heterosexual, coitocentrada y con fines reproductivos; una sexualidad encerrada en la pareja y el vínculo matrimonial, o la estigmatización de grupos de sexualidades no heteronormativas, entre otros. ${ }^{13}$

¿Qué consecuencia tiene esto? Inicialmente, si se supone que la reproducción de la especie humana es la finalidad de la sexualidad, entonces se infiere que la sexualidad legítima para ser enseñada es la heterosexual. De esta manera, se ignora e invisibiliza todo el resto de prácticas, experiencias y relaciones que son de orden también sexual: el placer, las prácticas de autodescubrimiento, las relaciones erótico-afectivas, amorosas o sexuales entre personas del mismo sexo, o personas heterosexuales que no desean reproducirse, por mencionar algunas. Como ha señalado Butler (2016), en su paso por la Argentina, "no todos los sujetos sexuados son reproductivos; hay personas que no tienen edad reproductiva, algunas personas jamás serán capaces de reproducirse, otros jamás querrán reproducirse, y muchos viven su vida sin reproducirse, entonces dada la gran multiplicidad de posiciones corpóreas en relación a este mandato cultural de reproducirse, ¿se puede decir que es necesario u obligatorio éticamente concebir al cuerpo sexuado fuera de los términos de la reproducción? Después de todo, la reproducción sexual es solo una manera de organizar y entender la sexualidad del cuerpo" (Butler, 2016, s/p).

En este contexto, la pedagogía queer nos invita a pensar lo impensado y afirmar "la precariedad del significado (...)" (Britzman, 2016, p.17). Allí, donde la palabra no alcanza, no llega, y el cuerpo se hace indecible, queda como alternativa "probar" en tanto que "juego alquímico que transmuta sentidos y haceres" (flores, 2017, s/p): 


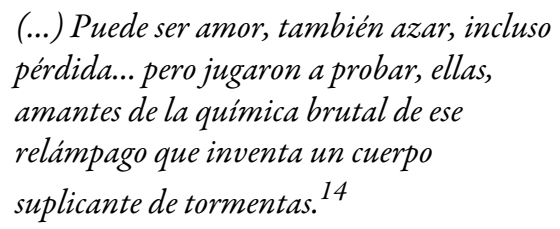

\section{La Pedagogización de la SEXUALIDAd o ¿QUÉ ES LO EDUCABLE EN LA EDUCACión SEXUAL?}

Si la sexualidad es algo que no solo muta a lo largo de los procesos históricos sino también en la vida misma de los sujetos, entonces, no podemos pensar este campo de experiencias como algo fijo o estable. De allí, la consideración sobre cierta imposibilidad de que la sexualidad (léase: el plano de los deseos de erotismo hacia otras personas) sea una dimensión a ser enseñada, al menos no plenamente. La historia de la educación sexual, por su parte, nos ha mostrado que la misma surge, en gran parte, de proyectos políticos y pedagógicos eugenésicos, participando en el establecimiento de jerarquías y desigualdades raciales, sociales y de género. Desde ya, si como referimos antes, son innegables los efectos de verdad de los discursos sobre la subjetividad y los cuerpos (el de los "expertos", el producido por los medios y la publicidad, los que circulan en la escuela), entonces estamos asumiendo que existe un poder performativo, configurador y regulador de los discursos y prácticas que son los marcos por los cuales nos asumimos como sujetos sexuados, como cuerpos generizados a partir de los regímenes de verdad y visibilidad que estén disponibles en aras de sostener las normas de género.

Frente a esto, la perspectiva de la pedagogía queer ha mantenido una mirada crítica sobre el propio término "educación sexual". Britzman (2002) sugiere que cuando la sexualidad ingresa en el currículum escolar, el lenguaje del sexo se torna -a los fines educativos- un lenguaje didáctico y explicativo y eso hace que se vuelva "des sexuado". A partir de allí, sugiere interrogar las relaciones entre educación y sexualidad, es decir, entre las relaciones escolares del conocimiento y el sexo, sobre todo, por considerar que este tiene un carácter inestable y abierto: “ ¿(...) puede ser el sexo educado y puede la educación ser sexuada? (...) Si el sexo es un tema inestable en sus objetivos, conocimientos, placeres y prácticas, ¿qué puede, entonces, ser exactamente dicho de él?" (Britzman, 2002, p. 231).

Esta problematización podemos advertirla, por ejemplo, en la Campaña Nacional por el Derecho al Aborto Legal, Seguro y Gratuito, lanzada en el año 2005, a partir de su consigna "Educación sexual para decidir, anticonceptivos para no abortar, aborto legal para no morir". ${ }^{15}$ Siendo la educación uno de sus nodos constitutivos, ¿qué podríamos decir, entonces, del sexo y la sexualidad? ¿Cómo imaginamos esa educación sexual? ¿Qué pedagogías contribuyen a la construcción de instancias y dimensiones que nos posibiliten decidir sobre nuestros cuerpos?

Diremos que aspiramos a una educación sexual en revisión permanente, inestable, contingente, precaria, atenta a las demandas de su tiempo y a lxs sujetos de la educación, en tanto sujetxs históricos y políticos. Pensadas desde la pedagogía queer, las pedagogías de la sexualidad quedan en este enfoque expuestas al cuestionamiento de sus tradiciones y perspectivas. Sus referentes han atendido a sus fundamentos preguntando cómo ciertos enfoques de enseñanza sobre la sexualidad y el género han sido configurados, cómo se regulan los cuerpos, cómo se los clasifican, qué se sabe de estos y, sobre todo, qué se ignora en las imbricadas relaciones entre conocimiento y poder (Britzman, 2002; flores, 2013; Lopes Louro, 1997, 1999).

En consonancia, pensar la enseñanza de la sexualidad desde la perspectiva pedagógica feminista tiene una potencia cuestionadora, transformadora y liberadora, en la medida que se busca la construcción de ambientes de enseñanza y aprendizaje que desborden las convenciones institucionales. Desde esa línea, se asumen temas como el aborto, ya no desde una tradición psicologista individual, sino como un tema colectivo, comunitario y, por tanto, profundamente político (Korol, 2007). También, desde una pedagogía del cuidado que enseñe 
sobre la autonomía corporal y la ciudadanía sexual más plena, para que los encuentros sexuales, eróticos y/o afectivos con otrxs sean más igualitarios, más libres y más placenteros.

Aspiramos a una educación sexual que acoja los llamados de los bordes y cante con las Krudas Cubensi: ${ }^{16}$

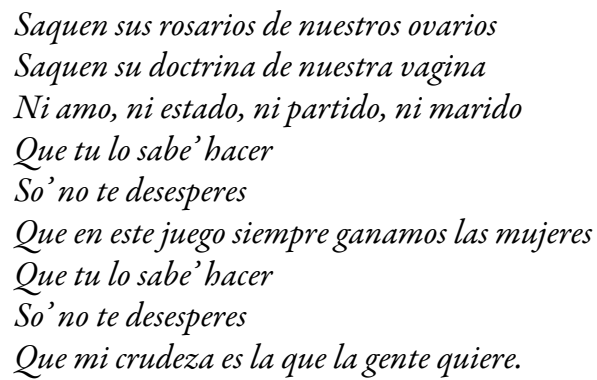

\section{LA MIRADA ADULTOCÉNTRICA DE LA SEXUALIDAD o ¿CÓMO APRENDEMOS A SER NIÑOS O} NIÑNA?

Es paradójico en un punto que, si bien en términos generales se ha considerado desde hace mucho tiempo la idea de que la infancia es asexuada, al mismo tiempo, ya se sabe, la escuela cotidianamente motoriza fuertes discursos normalizadores del género sobre el comportamiento, la vestimenta, los gestos, los modos de hablar, de caminar, de jugar y bailar de lxs nińxs. Es decir, aun cuando se reconoce que la sexualidad corresponde a un proceso que se inicia en la pubertad (idea fuertemente difundida en psicólogos y pedagogos modernos como Víctor Mercante), la escuela trabaja con discursos y prácticas sobre las normas de género y sexualidad otorgando valoraciones al comportamiento, los modos de actuar y de ser: "No te sientes como un varón", "No grites como las nenas", son algunas de estas expresiones. Las nombradas "nuevas infancias" rompen con su concepción moderna (obediencia, docilidad, minoría de edad, inocencia, fragilidad, dependencia), no solo por el reconocimiento de derechos a lxs niñxs, sino también porque exigen mayores condiciones legales, normativas, políticas, jurídicas y culturales para las decisiones que les involucre.

Por ejemplo, para la decisión sobre el propio cuerpo y la experiencia de género, podemos referir a las "infancias trans": sujetxs niñxs que no se identifican desde temprana edad con su género asignado. En la Argentina, ha sido muy conocida la historia de Luana, una niña trans que, con ayuda de su madre, logró su cambio de identificación en su documento nacional de identidad, ${ }^{17}$ experiencia que ha servido para la lucha contra la violencia institucional hacia las infancias trans. ${ }^{18}$

Aun cuando asumamos sin dudas que, tanto lxs niñxs como lxs jóvenes, son sujetos en formación, que están "siendo", como adultxs deberíamos cesar la tendencia a clasificar los comportamientos, o a hacer observaciones sobre el modo de actuar, de hablar o de ser de los más jóvenes en relación con sus deseos y experiencias en relación a las identificaciones genéricas que vayan experimentando. Esto conlleva a suspender ciertos juicios o mitos sobre el comportamiento de lxs niñxs en sus expresiones generizadas, con que se establecen vínculos entre presente y futuro, en torno a los juegos, los colores, las actividades y oficios, entre otros. Sostiene Preciado (2009, p. 165) que "la infancia no es un estadio pre-político sino, por el contrario, un momento en el que los aparatos biopolíticos funcionan de manera más despótica y silenciosa sobre el cuerpo”. Romper con la idea moderna de que la niñez es asexuada y que se encuentra vacía de experiencia, es otorgarle un lugar para el propio autocuestionamiento adultocéntrico.

Refiriéndose a Luana, la activista trans Susy Shock admite que:

Nosotras fuimos y somos niñas a través de Lulu y tantísimes otres que nos están arrastrando con urgencia a la conciencia que el abrazo es ya, no mañana. Que el futuro es demasiado espeso y lejano si lo que guía es solo el ejemplo de esa inercia binaria. Que las tías travas no queremos ser como reza nuestra teoría y poética transtrava latinoamericana ni Mirtha Legrand ni Valeria Mazza y huimos traidoras sinérgicas de ser Rambo o el príncipe azul. Sobre todo que nos seguimos creando y 
deconstruyendo a cada paso para aprender de esa juventud que nos dice que lo no binario es un enorme lugar para animarse a enchastrarse y explorar, que las respuestas las vamos creando en cada cuerpo y deseo y que eso, finalmente, es enormemente político y sagrado (Shock, 2018, video).

\section{A Modo de CIERRE (IN)ESTABLE}

Pensar una pedagogía queer significa, entonces, aceptar que los cuerpos, las sexualidades, los deseos, las sensibilidades, las emociones no pueden sustraerse al juego de la significación y de la política. Pensar en queer significa rechazar al dimorfismo sexual (macho/hembra), para develar que es solo una construcción cultural más. Pensar en clave queer implica rechazar la heterosexualidad como la matriz o régimen normal de deseo y placer. Pensar en clave queer implica deconstruir la topografía corporal falologocéntrica. Pensar en clave queer implica cuestionar el fundamento que presupone que las identidades son auto-idénticas, persistentes a través del tiempo, unificadas e internamente coherentes. Pensar en clave queer presupone que los cuerpos no son un datobiológico, universal y sin historia, sino una construcción cultural discursiva y performativa más. Pensar una pedagogía queer es, finalmente, animarse a reflexionar y a sentir qué otras maneras, prácticas y significados son posibles.

La escuela es un espacio de formación política en el que podemos denunciar el régimen heterosexual sobre el que se ha fundado la idea misma de educación, de nación, unos modos correctos de ser y estar, el rechazo de otros modos... Deconstruir el régimen político-erótico implica también deconstruir la escuela, las prácticas pedagógicas, los saberes escolares, la cultura escolar, el lugar de lxs maestrxs, la atención que le prestamos a la educación y a la pedagogía. Es importante también -y urgente- nutrir este análisis mirando experiencias pedagógicas que arrojen otros elementos: prácticas pedagógicas de resistencia, legitimación de otros saberes, en términos de Britzman aspiramos a "albergar las preguntas más incómodas, las cuestiones que perturban, erotizar los procesos de conocer, de aprender y de enseñar, dando lugar al placer y a las curiosidades impertinentes" (Morgade, 2009, p. 39)

Seguir una pedagogía queer, en diálogo con las pedagogías feministas, son puntos de partida epistemológicos, políticos y pedagógicos, y sólo pueden ser inestables, contingentes, plurales, contradictorios, ambiguos y afirmativamente históricos. Reconocemos a través de ellos, en primer término, la importancia del lenguaje, los discursos y las relaciones de poder a la hora de conceptualizar, significar y producir efectos en los cuerpos, sexualidades, deseos, sensibilidades y placeres. Todos estos son constructos epistémicos, productos de regímenes de verdad situados espacial e ideológicamente, interesados y fuertemente epocales; mediados por las culturas, los rituales, las creencias, los valores, los usos y costumbres, y los saberes.

\section{REFERENCIAS}

Agencia Presentes (2017). Besos Lesbianos. Procesan a Mariana Gómez, detenida cuando besaba a su esposa. (fotografía). Recuperado de: http://agenciapresentes.org/2018/02/02/besoslesbianos-procesan-mariana-gome z-detenida-besar-esposa/

Baden-Powell, R. (1998 [1908]). Escultismo para Muchachos. San José de Costa Rica: Editorial Scout Interamericana. Badinter, E. (1993). XY La identidad masculina. Madrid: Alianza.

Berlant, L. y Warner, M. (2002). Sexo en público (pp. 229-257). En R. Mérida (ed.), Sexualidades transgresoras. Una antología de los estudios queer. Barcelona: Icaria.

Boccardi, F. (2008). Educación sexual y perspectiva de género. Un análisis de los debates sobre la ley de educación sexual integral en Argentina. Revista perspectivas de lacomunicación, 1(12), 48-58.

Britzman, D. (2002). La pedagogía transgresora y sus extrañas técnicas (pp. 197-228). En R. Mérida (ed.), Sexualidades transgresoras. Una antología de los estudios queer. Barcelona: Icaria. 
Britzman, D. (2016). ¿Hay una pedagogía queer? O, no leas tan recto. Revista de educación, 7(9), 13-34. Recuperado de http://fh.mdp.edu.ar/revistas/index.php/r_educ/issue/view/110

Butler, J. (2009). Lenguaje, poder e identidad. Madrid: Síntesis.

Butler, J. (2016) "Cuerpos que aun importan". Conferencia dictada en la Universidad Nacional de Tres de Febrero, Argentina. Recuperado de https://www.youtube.com/watch?v=-UP5xHhz17s\&t=5250s

Caruso, M. y Dussel, I. (1996). “Modernidad y escuela: los restos del naufragio” (pp. 89-103). En M. Caruso y I. Dussel, De Sarmiento a los Simpons. Cinco conceptos para pensar la educación contemporánea. Buenos Aires: Kapelusz.

Connell, R. (Robert) (1998). El imperialismo y el cuerpo de los hombres (pp. 76-89). En T. Valdés y J. Olavarría (eds.), Masculinidades y equidad de género en América Latina.Santiago de Chile: FLACSO.

Da Silva, T. T. (1999). Documentos de identidad. Una introducción a las teorías del currículum. Belo Horizonte: Autentica Editorial.

Draghi, M. J. y Vassilliades, A. (2014). Presentación a la reedición del artículo: "La Facultad de Ciencias de la Educación" de Víctor Mercante. Archivos de ciencias de la educación, 8 (8), 1-3. Recuperado de http://www.m emoria.fahce.unlp.edu.ar/art_revistas/pr.6599/pr.6599.pdf

Dussel, I. (2013). "Presentación. Víctor Mercante: La adolescencia como categoría escolar. La emergencia de una problematización” (pp. 11-42). En V. Mercante, La crisis de la pubertad y sus consecuencias pedagógicas, La Plata: UNIPE-Editorial Universitaria.

Espinoza Miñoso, Y. (2008). Dislocando saberes y prácticas de la perspectiva de género en la educación: de la búsqueda de equidad a la fractura del sujeto educativo. Recuperado de https://www.academia.edu/1097680/Dislocando_saberes_y_pr\%C3\%A1cticas_de_la_perspectiva_de_g\% C3\%A9nero_en_la_educaci\%C3\%B3n_de_la_b\%C3\%BAsqueda_de_equidad_a_la_fractura_del_sujeto_ed ucativo

flores, v. (2013). Interruqciones. Ensayos de poética activista escritura, politica, pedagogía. Neuquén: Editora La Mondonga Dark.

flores, v. (2016). Afectos, pedagogías, infancias y heteronormatividad. Reflexiones sobre el daño (pp. 13-30). En Pedagogias transgresoras. Córdoba: Bocavulvaria.

flores, v. (2017). Jugaron a probar. Performance realizada con Fer Guaglione el 5 de noviembre de 2017 en Transmutaciones vol2, La Plata (ARG). Recuperado de http://escritoshereticos.blogspot.com

Foucault, M. (1999). El orden del discurso. Barcelona: Tusquets.

Foucault, M. (2002). La arqueologia del saber. Buenos Aires: Editorial Siglo XXI.

Hantover, J. (1978). The boy scouts and the validation of masculinity. Journal of social issues, 34(1), 184-195.

Jones, D. (2009). ¿De qué hablamos cuando hablamos de sexualidad? Educación sexual en escuelas de nivel secundario antes de la Ley de Educación Sexual Integral de la Argentina. Argumentos. Revista de crítica social, 11, 63-82.

Korol, C. (comp.) (2007). Hacia una pedagogía feminista. Buenos Aires: El Colectivo, América Libre.

Lemebel, P. (1986). "Manifiesto (Hablo por mi diferencia)". Texto leído como intervención en un acto político de la izquierda en septiembre de 1986, Santiago de Chile.

Lopes Louro, G. (1997). Gênero, sexualidade e educação. Uma perspectiva pós-estruturalista. Petrópolis: Voces.

Lopes Louro, G. (1999). Pedagogías da sexualidade (pp. 7-34). En O corpo educado. Pedagogias da sexualidade. Belo Horizonte: Autêntica.

Magdalena, L. (2017). Ningún beso nace hetero (fotografía). Recuperado de http://reviradas.com.ar/2017/10/07/b ezaso-en-constitucion/.

Mansilla, G. (2014). Yo nena, yo princesa. Luana, la niña que eligió su propio nombre. Buenos Aires: Universidad Nacional General Sarmiento.

Méndez, M. y Scharagrodsky, P. (2016). El Escautismo en la Argentina: los diferentes sentidos sobre la naturaleza y la vida al aire libre a principios del siglo XX (pp. 113-139). En C. Soares (coord.), Uma educação pela natureza: a vida ao ar livre, o corpo e a ordem urbana. Sao Paulo: Editora Autores Associados. 
Mercante, V. (2013 [1918]). La crisis de la pubertad y sus consecuencias pedagógicas. La Plata: UNIPE, Editorial Universitaria.

Ministerio de Cultura y Educación (1992). Sexualidad. Educación de la sexualidad. Documentos de apoyo para la educación integral. Serie Documentos y Problemas Sociales. Buenos Aires: Ministerio de Cultura y Educación.

Ministerio de Educación y Justicia (1987). Educación sexual. Series Demandas de información educativa. Buenos Aires: Secretaria de Educación. Centro Nacional de Información, Documentación y Tecnología Educativa.

Morgade, G. (2009). Educación, relaciones de género y sexualidad: caminos recorridos, nudos resistentes. (pp. 19-50). En A. Villa, Sexualidad, relaciones de género y de generación. Perspectivas históricos-culturales en educación. Buenos Aires: Novedades Educativas.

Pineau, P. (2014). A modo de introducción. Estética escolar: manifiesto sobre la construcción de un concepto (pp. 21-35). En P. Pineau (Dir.),Escolarizar losensible. Estudios sobre estética escolar, 1870-1945. Buenos Aires: Teseo.

Preciado, B. (Paul) (2009). Terror anal: apuntes sobre los primeros días de la revolución sexual (pp. 135-174). En G. Hocquenghem, El deseo homosexual. Barcelona: Melusina.

Preciado, B. (Paul) (2014). Sín título. Ponencia presentada en Cuerpos inapropiables propiedad, expropiación y políticas de lo «común». Recuperado de http://www.macba.cat/es/audio-beatriz-preciado-cuerpos-inapropia bles

Rich, A. (1999). La heterosexualidad obligatoria y la existencia lesbiana. En M. Navarro y C. Stimpson (comp.), Sexualidad, género y roles sexuales. (pp. 159-211). Buenos Aires: F.C.E.

Salessi, J. (1995). Médicos maleantes y maricas. Higiene, criminologia y homosexualidad en la construcción de la nación argentina. (Buenos Aires: 1871-1914). Rosario: Beatriz Viterbo.

Scharagrodsky, P. (2004). El scautismo en la educación física bonaerense argentina o acerca del buen encauzamiento varonil (1914-1916). Mora, 9/10, 50-66.

Scharagrodsky, P. (2007). Masculinidades valuadas y devaluadas. Tensiones, límites y posibilidades en el ámbito escolar (pp. 263-284). En R. Baquero, G. Frigerio y G. Diker, Las formas de lo escolar. Buenos Aires: Del Estante.

Shock, S. (2018). Intervención en la presentación del libro Mariposas libres. El derecho a vivir una infancia trans. Recuperado de https://www.youtube.com/watch?v=fd24Utyfas8.

Talak, A. M. (2010). Progreso, degeneración y darwinismo en la primera psicología Argentina, 1900-1920 (pp. 299-320). En G. Vallejo y M. Marida (Dir.), Derivas de Darwin. Cultura y política en clave biológica. Buenos Aires: Siglo XXI.

Theumer, E. (2018). Lenguaje incisivo. Blog Parole de queer. Recuperado de http://paroledequeer.blogspot.com/20 18/09/lenguaje-incisivo-emmanuel-theumer.html

Tin, L. G. (2012). La invención de la cultura heterosexual. Buenos Aires: El Cuenco del Plata.

Torres, D. (2011). Pornoterrorismo. Tafalla: Editorial Txalaparta.

Trujillo, G. (2015). Pensar desde otro lugar, pensar lo impensable: hacia una pedagogía queer. Educação e pesquisa, 41, 1527-1540. Recuperado de: http://www.scielo.br/pdf/ep/v41nspe/1517-9702-ep-41-spe-1527.pdf

Wainerman, C., Di Virgilio, M. M. y Chami, N. (2008). La escuela y la educación sexual. Buenos Aires: ManantialUNFPA.

Wittig, M. (2006). El pensamiento heterosexual y otros ensayos. Madrid: Egales.

Zemaitis, S. (2016). Pedagogias de la sexualidad. Antecedentes, conceptos e historia en el campo de la educación sexual de la juventud. Trabajo Final de Especialización en Nuevas Infancias y Juventudes, Facultad de Humanidades y Ciencias de la Educación de la Universidad Nacional de La Plata, La Plata.

Zemaitis, S. (En prensa). Educación sexual. En F. Fiorucci y J. Bustamante Vismara (Eds.), Historia de la educación argentina: palabras claves. Buenos Aires: Editorial UNIPE. 


\section{Notas}

1 Lxs autores agradecen los aportes de lxs lectorxs y evaluadorxs del artículo por enriquecerlo con sus sugerencias y preguntas críticas. Agradecemos especialmente a val flores por sus valiosos comentarios del primer borrador de este trabajo.

2 Proyecto acreditado por la Universidad Nacional de Quilmes (UNQ) (CS n ${ }^{\circ}$ 623/15) con vigencia hasta 2018. Dirigido por el Dr. Pablo Scharagrodsky, cuenta con la participación de estudiantes y docentes de diferentes disciplinas de la UNQ y la Universidad Nacional de La Plata (UNLP). El propósito es la sensibilización y concientización sobre las problemáticas que atañen a las personas LGBTIQ+, a través de la realización de talleres sobre temáticas de diversidad y desobediencia sexo-genérica desde una perspectiva feminista y queer. Se realizan entre tres y cuatro talleres por semestre, con los grupos de estudiantes de los profesorados en Trabajo Social, Psicología y Psicopedagogía, de un Instituto de formación docente ubicado en la localidad de Quilmes. Su diseño y selección de temas, se trabajó en la primera fase del proyecto, mediante reuniones del equipo de extensión y la posterior aprobación de las directivas del instituto. Al finalizar cada semestre, se realiza una evaluación de las jornadas de la cual surgen los aspectos a mejorar o ajustar para su implementación en el semestre siguiente. La sistematización por parte del equipo se realiza a través de actas de cada encuentro.

3 El uso de la " $\mathrm{x}$ " en este texto no responde a un posicionamiento en el marco de la inclusión y la diversidad, sino que pretende acoger la provocación de Yuderkys Espinoza Miñoso (2008), como tachado e impugnación de la intencionalidad genérica. Se trataría "de abandonar la empresa de hacer incluir en ellos todo lo que le excede, abandonar la idea de la necesidad del género para pensarse, para existir” (p. 1). Emmanuel Theummer (2018), por su parte, dirá que el uso de la "x" y la "e" evidencian, precisamente, la indecibilidad del género o la imposibilidad de reducirlo a categorías estables. Por otro lado, es bien conocido el rechazo por parte de instituciones como la Real Academia Española frente al uso de signos o letras que pretendan incluir o ampliar las limitaciones de la lengua, que lo considera innecesario, ya la inclusión la ofrecería el masculino genérico; además, descalifica estas acciones, por considerarlas prácticas de feministas y promotores de "la ideología de género". En ese sentido, la "x" también estaría dotada de incomodidad e irreverencia frente a las reglas que hacen del lenguaje un imperativo. Como sugiere Judith Butler (2009, p. 73): "La resignificación del lenguaje requiere abrir nuevos contextos, hablando de maneras que aún no han sido legitimadas, y por lo tanto, produciendo nuevas y futuras formas de legitimación". No obstante ello, el desarrollo de algunos apartados nos llevaba de vuelta a resoluciones que apelaban necesariamente al binarismo, principalmente aquellos que requirieron que la reflexión se hiciera desde el referente de los derechos civiles. Esto por ser la única vía, hasta ahora, que permite garantizar la inclusión de identidades que requieren una visibilidad inmediata. Nos enfrentamos a una tensión, entre cuestionar los fundamentos de la pedagogía moderna que es constitutivamente binarista, cuidar algunas de las apuestas ya existentes (como el enfoque diferencial, la legislación incluyente o la misma ESI - educación sexual integral) y los fundamentos de la pedagogía queer, para la cual, la misma inclusión resulta un encerramiento identitario.

4 Entendemos pedagogía queer tomando los aportes de la militante y docente argentina valeria flores y del pedagogo brasileño Tomaz Tadeu Da Silva. Para flores, la pedagogía queer es un movimiento que "pretende leer en forma compuesta las diversas estructuras de sujeción y opresión que determinan y condicionan socialmente las identidades", y su crítica, que es al mismo tiempo política, sexual y pedagógica, gira en torno a la reflexión de los significados sobre las relaciones entre saber/poder, las sexualidades y el género. Según su definición, una pedagogía antinormativa hace el trabajo de deconstruir la educación como una de las formas de control de la vida sexual al mismo tiempo que promueve la no jerarquización de deseos o identidades codificados por una "epistemología patologicista de las identidades" (flores, 2013, p.240). Tadeu Da Silva, por su parte, propone una pedagogía queer que no se limite a incluir en el currículum informaciones 'correctas' y aceptadas como científicas sobre la sexualidad, sino que se trate de una pedagogía que cuestione los procesos institucionales y discursivos, las estructuras de significación que definen qué es lo correcto y qué lo incorrecto, qué es lo moral y qué lo inmoral, lo deseable y lo indeseable, lo normal y lo anormal (Da Silva, 1999).

5 Entendemos pedagogía feminista, como una corriente de pensamiento pos-crítico sobre la educación, que desde el análisis de sus condiciones históricas, sociales y políticas; problematiza los saberes que le sustentan, convengamos, relativos al conocimiento, los sujetxs de la educación, la transmisión, entre otros. Su reflexión, principalmente epistemológica, introduce cuestionamientos al entramado disciplinar que conforma el currículo escolar, produciendo prácticas, representaciones, formas de conocer y asumirse en el mundo, que provienen de una epistemología dominante que es androcéntrica, binarista, racista, clasista y heteronormativa (Da Silva, 1999; Korol, 2007). En relación a los estudios de género, como se advierte en el texto, no estaríamos hablando de un concepto, sino de un conjunto de estudios que producen categorías analíticas teóricas de las cuales nos servimos para deconstruir la educación de la sexualidad.

6 Los objetivos de la Ley 26.150, sancionada en Argentina el 4 octubre de 2006 bajo el nombre de Programa de Educación Sexual Integral, son 1. Incorporar la educación sexual integral dentro de las propuestas educativas orientadas a la formación armónica equilibrada y permanente de las personas. 2. Asegurar la transmisión de conocimientos pertinentes, 
precisos y confiables y actualizados sobre los distintos aspectos involucrados en la educación sexual integral. 3. Promover actitudes responsables ante la sexualidad. 4. Prevenir los problemas relacionados con la salud en general y la salud sexual y reproductiva en particular. 5. Procurar igualdad de trato y oportunidades para mujeres y varones.

7 "Razón”, “ciencia”, “orden”, "libertad” e "igualdad” fueron los principales pilares ideológico-políticos mediante los cuales se sostendrían las nuevas sociedades modernas (en contraposición con la centralidad de Dios, de la fe y de la divinidad como principios organizadores del tejido y las jerarquías sociales). Los ciudadanos -varones-, libres e iguales, portadores de derechos y obligaciones ante la ley (o al menos en términos de proyección política) debían ser formados como tales por los Estados-nación a los fines del cumplimiento de la vida pública. Es allí donde vemos aparecer la función social y política de la educación, o más precisamente, el interés moderno de los Estados en crear instituciones masivas que forman estos ciudadanos. Así, la educación como interés de Estado comenzó en el siglo XIX, cuando las sociedades modernas, con sus Estados-nación en consolidación, necesitaron de un lugar, una institución, que pueda educar a la masa de niños y jóvenes, convirtiéndose el sistema educativo en un eje central del proyecto moderno-liberal.

8 Es importante aclarar que la cita que utilizamos, busca visibilizar las operaciones de patologización y criminalización de la homosexualidad. Asimismo, queremos señalar que nuestra postura ético-política no admite ciertas prácticas sexuales como, por ejemplo, la pedofilia o la zoofilia ya que en sí mismas son prácticas violentas, coercitivas, cosificantes y de franca dominación.

9 Conservamos la palabra "homosexualidad" en la enunciación de este apartado por ser la que más presencia ha tenido en el pensamiento moderno y es la que figura en el ejemplo que tomamos el cual, como veremos, incluía al lesbianismo. No obstante, autoras como Adrienne Rich (1999) expondrán que tales denominaciones invisibilizaban la existencia lesbiana, mostrándolas como réplicas de las heterosexuales u homosexuales masculinas. Para el presente, es necesario enfatizar en su singularidad, por ello la enunciamos diferenciada en el desarrollo.

10 Nos referimos a la sanción de una serie de leyes y normativas como la ley de Salud Sexual y Procreación Responsable (25.673) y el desarrollo de su Programa Nacional, la ley de Identidad de Género (26743), la Ley de Matrimonio Civil entre parejas del mismo sexo (26.618), la ley de Prevención y sanción de la trata de personas y asistencia a sus víctimas (26.364), la ley de Protección integral para prevenir, sancionar y erradicar la violencia contra las mujeres (26.485), como así también la reciente incorporación del femicidio al Código Penal.

11 Sobre el concepto de 'emoción fiamesca' utilizada por Mercante, Inés Dussel (2013) señala que esta es una expresión propia de esa época. La autora rastrea esta expresión en un texto posterior al de Mercante, Ambición y angustia de los adolescentes, del pedagogo argentino Aníbal Ponce. En este texto publicado por primera vez en 1936, Ponce hace referencia a "los afectos equívocos" en la adolescencia y "que los psicoanalistas no vacilan en clasificar de homosexual, para el cual muchos idiomas tienen palabras especiales: fiamma en italiano, flammes en francés (...)" (Ponce, 1947, citado en Dussel, 2013, p.27).

12 La descripción del homosexual como uranista (en alemán: urning) la hizo en 1862 un primer activista alemán, Kart Heinrichs Ulrichs basándose en el discurso de Pausanías en el Simposio de Platón. En ese texto, Pausanías describió el amor celestial inspirado en Urania, la musa de los uranistas u hombres que amaban a otros hombres" (Salessi, 1995, p. 225).

13 Pero, al lado de esto, fue insistente en la promoción de la autonomía de las mujeres para el control de su fertilidad, el cuestionamiento a los prejuicios en la sexualidad, particularmente en lo que tiene que ver con el uso de los métodos de anticoncepción, y el trabajo con un cuerpo médico que pudiera comprender al individuo en su integralidad, y no desde sus síntomas.

14 Poema de val flores "Jugaron a probar". Usado en la performance realizada con FerGuaglione el 5 de noviembre de 2017 en Transmutaciones vol 2, La Plata (ARG). El registro audiovisual y texto completo en el blog http://escritosheretico s.blogspot.com

15 La Campaña Nacional por el Derecho al Aborto Legal, Seguro y Gratuito "es una amplia y diversa alianza federal, que articula y recupera parte de la historia de las luchas desarrolladas en nuestro país en pos del derecho al aborto legal, seguro y gratuito". A partir del año 2004, en los Encuentros Nacionales de mujeres, se discutió la necesidad de impulsar un conjunto de acciones y estrategias, para defender el derecho al aborto y la autonomía de los cuerpos gestantes, como cuestión de salud pública, justicia social y de derechos humanos. Trabajan en esta, de manera mancomunada: grupos feministas, movimientos políticos y sociales, organizaciones y personalidades vinculadas a los derechos humanos, la academia, la cultura, redes educativas, entre otros. Fue lanzada el 28 de mayo de 2005, Día de Internacional de Acción por la Salud de las Mujeres. Toda la información sobre la campaña en http://www.abortolegal.com.ar/

16 Banda de hip hop activista y feminista (feminismo negro y queer) que surge en Cuba a finales de los noventa, conformado por Odaymara Cuesta y Olivia Prendes. La canción referenciada se titula "Mi cuerpo es mío". El video se puede ver en: https://www.youtube.com/watch?v=x-Pgwldfx $8 U$

17 La historia de Luana fue narrada y dada a conocer por su mamá quien escribió en forma de memorias personales todos los obstáculos que ambas debieron atravesar hasta lograr el reconocimiento total de la nueva identidad de la niña.

18 Este relato puede leerse en Mansilla (2014). 